\title{
Identification of critical microRNAs in gastrointestinal stromal tumor patients treated with Imatinib
}

\author{
Z. ZHANG ${ }^{1}$, N. Y. JIANG ${ }^{2}$, R. Y. GUAN ${ }^{2}$, Y. K. ZHU' 1 , F. Q. JIANG ${ }^{3}$, D. PIAO ${ }^{1, *}$ \\ ${ }^{1}$ Department of Colorectal Surgery, The First Affiliated Hospital of Harbin Medical University, Harbin, China; ${ }^{2}$ Respiratory Medicine, Infectious \\ Disease Hospital of Heilongjiang Province, Harbin, China; ${ }^{3}$ General Surgery, Heilongjiang Provincial Hospital, Harbin, China
}

*Correspondence: DaxunpiaoPDX@163.com

Received September 6, 2017 / Accepted January 5, 2018

\begin{abstract}
Gastrointestinal stromal tumors (GISTs) are the most common mesenchymal neoplasms of the gastrointestinal tract. Imatinib mesylate was considered a breakthrough drug in clinical treatment of GIST, but GIST patients showed resistance to it. We aimed to identify critical microRNAs (miRNAs) related to imatinib resistance in imatinib-treated GIST patients. Microarray datasets under accession numbers GSE63159 and GSE45901 were downloaded from the Gene Expression Omnibus (GEO) database. The differentially expressed miRNAs (DEMs) related to imatinib resistance were identified. GO function and KEGG pathway enrichment analyses were performed, and lncRNA-miRNA-target gene regulatory networks were constructed. Finally, the critical miRNAs and their target genes related to imatinib resistance or sensitivity were identified. A total of 20 DEMs in the GSE63159 dataset (7 significantly up-regulated and 13 down-regulated) and 23 DEMs in the GSE45901 dataset ( 8 up-regulated and 15 down-regulated) were identified. Five critical miRNAs and 109 target genes were identified in the lncRNA-miRNA-target gene regulatory networks. GO function and KEGG pathway enrichment analysis showed that target genes of DEMs were mainly involved in several signaling pathways, such as focal adhesion and the GnRH signaling pathway. From the five miRNAs, the overexpression of $h s a-m i R-28-5 p$ and $h s a-m i R-125 a-5 p$ had significant correlation to imatinib resistance or imatinib sensitivity in GIST patients. In addition, Hsa-miR-28-5p and $h s a-m i R-125 a-5 p$ may be involved in the development and progression of GIST, and they may serve as prognostic markers for imatinib-response in GIST patients.
\end{abstract}

Key words: gastrointestinal stromal tumors, microRNAs, imatinib mesylate, RNA, long noncoding, focal adhesions, gonadotropin-releasing hormone

Gastrointestinal stromal tumors (GIST) are the most common mesenchymal neoplasm of the gastrointestinal tract, with an incidence of 1-2 cases/100,000 individuals/ year worldwide [1]. Most GIST patients express mutation of two key oncogenic genes, v-kit Hardy-Zuckerman 4 feline sarcoma viral oncogene homolog (KIT) or platelet-derived growth factor receptor a (PDGFRA) [2, 3]. Today, imatinib mesylate is specifically applied in clinical settings to target KIT and PDGFRA encoded proteins, and it is considered a breakthrough drug in GIST treatment $[4,5]$. Although the clinical application of imatinib has improved outcomes for patients, drug resistance still remains an urgent challenge for GIST therapy.

microRNAs (miRNAs) are small noncoding RNAs with 22 nucleotides and they control tumor cell growth by regulating the expression of multiple gene products and the function of cellular pathways [6]. It is recently reported that miRNAs promote or inhibit tumor growth effects in several kinds of cancers and they have therefore been identified as targets for cancer therapy, diagnosis, and prognosis [7, 8]. Most studies on GIST focus on the effects of miRNA expression on tumorigenesis [9], overall patient survival [10] or KIT and PDGFRA mutations [11]. Although miRNAs have been reported as vital regulators in KIT and PDGFRA expression, the functional role of miRNAs in the imatinib-response in GIST patients has rarely been reported [12].

In 2014, Akcakaya et al. [13] performed analysis of miRNA expression profiles to investigate the miRNA expression signatures related to the imatinib-response and KIT mutational status in GIST patients. They found that $m i R-125 a-5 p$ and its target gene, tyrosine-protein phosphatase non-receptor type (PTPN18), played an important role in imatinib resistance in GIST cells. Herein, we used the microarray data deposited by Akcakaya et al. to identify critical miRNAs, target genes, and 
potential regulatory pathways related to imatinib resistance in GIST samples. In addition, Gene Ontology (GO) function and Kyoto Encyclopedia of Genes and Genomes (KEGG) pathway enrichment analyses were performed. The related lncRNAs of miRNAs were searched, and a competing endogenous RNA (ceRNA) regulatory network was constructed. The objective of this study was to identify critical miRNAs related to imatinib resistance which could serve as important therapeutic targets or prognostic biomarkers in GIST.

\section{Materials and methods}

Data preprocessing and differentially expressed miRNA (DEM) screening. Two microarray datasets under accession numbers GSE63159 and GSE45901[13], including 34 and 17 GIST human samples respectively, were downloaded from the NCBI (National Center for Biotechnology Information) CEO (Gene Expression Omnibus) database (https://www. ncbi.nlm.nih.gov/). GSE63159 included 19 GIST patient samples which were treated with imatinib and 15 untreated samples and GSE45901 included 17 GIST samples treated with imatinib. Seven of these samples were imatinib-resistant and ten were imatinib-sensitive. The two microarray datasets were analyzed based on the GPL10656 Affymetrix Human Gene 1.0 ST Array platform.

The Limma package [14] in $\mathrm{R}$ software (version 3.4.1) normalized the gene expression profile. The t-test (http://127.0.0.1:26738/library/stats/html/t.test.html) and Wilcoxon rank sum test (http://127.0.0.1:26738/library/ stats/html/wilcox.test. html) then screened the DEMs related to GIST progression in R software (3.4.1 version). DEMs in the GSE63159 dataset were screened in the imatinib-treated GIST samples and compared to untreated samples. DEMs in the GSE45901 dataset were then screened from imatinib-sensitive GIST samples and compared to imatinib-resistant samples. Therefore, the DEMs with the cut-off criteria of a logFC greater than 0.5 and p-value less than 0.05 were selected. Finally, we used the intersection of the DEMs from the t-test and Wilcox-test methods, and Venn diagrams visualized the results.

Bidirectional hierarchical clustering analysis. After microarray data from the GSE63159 and GSE45901 datasets were preprocessed, the expression values of DEMs in the two groups were extracted and converted into the expression matrix using the Affy package [15]. The pheatmap package [16] in R software (3.4.1 version) analyzed the expression matrix values based on the Euclidean distance [17]. Bidirectional hierarchical clustering analysis was then performed and gene expression values were displayed in heat maps $[18,19]$. Similar gene expression values could be clustered together based on the bidirectional hierarchical clustering analysis.

Construction of miRNA co-expression network and miRNA-target genes network. The $\mathrm{R} 3.4 .1$ software (http://127.0.0.1:20874/library/stats/html/cor.html) calcu- lated the Pearson correlation coefficient between two DEMs obtained from the GSE63159 dataset; resultant miRNAs with a Pearson correlation coefficient greater than 0.8 were obtained. The miRNA-miRNA co-expression network was constructed. Target genes that were co-expressed with miRNAs were searched in three miRNA-target gene databases: miRanda [20] (http://www.microrna.org/microrna/home. do), miRTarBase 2016 [21] (http://mirtarbase.mbc.nctu.edu. tw/), and TargetScan release 7.1 [22] (http://www.targetscan. org/). We found intersections between the miRNAs and target genes in the three databases and constructed the regulatory network. Finally, the complex miRNA-target gene network was visualized by Cytoscape 3.4.0 software [23] (http://www. cytoscape.org/).

GO and KEGG pathway analysis. DAVID 6.8 performed the GO function and KEGG pathway enrichment analysis based on the GSE63159 dataset. A p-value less than 0.05 was the threshold, and the formula for calculating the p-value is as follow:

$$
p=1-\sum_{i=0}^{H-1} \frac{\left(\begin{array}{c}
M \\
1
\end{array}\right)\left(\begin{array}{l}
N-M \\
K-H
\end{array}\right)}{\left(\begin{array}{l}
N \\
K
\end{array}\right)}
$$

where $N$ is the number of genes that have GO functional annotation, $K$ is the number of DEMs in $N$, and $M$ is the number of gene corresponding to a special GO function node.

Predicting the risk lncRNAs of miRNAs. The risk lncRNAs regulated by the five DEMs obtained from the GSE63159 dataset (hsa-miR-324-5p, hsa-miR-342-3p, $h s a-m i R-28-5 p$, hsa-miR-125a, and hsa-miR-320a) were searched using the starBase V2.0 database which is derived from 37 independent research centers, and it contains more than 6000 samples from 14 types of cancers. The interactions between lncRNAs, circRNAs, proteins and miRNAs were analyzed using this database. The relationship between miRNA-target genes and the IncRNA-miRNA-target gene network, also called the ceRNA network, was constructed and Cytoscape3.4.0 (http://www.cytoscape.org/) visualized the regulatory networks.

Analysis of critical genes related to imatinib resistance. The important DEMs related to imatinib resistance or imatinib sensitivity were analyzed based on the GSE45901 dataset. After integrating the DEMs found in the GSE63159 dataset, several critical DEMs related to imatinibresponse were obtained. By combining these DEMs with the miRNAs-target gene regulatory network, we performed the GO function and pathway enrichment analysis to further explore the key functions of these critical DEMs.

\section{Results}

Data preprocessing and DEMs screening. The microarray data in the GSE63159 and GSE45901 datasets were normalized and the results are shown in Figure 1 (A and B). 
After data preprocessing, a total of 21 DEMs from each set in the imatinib-treated samples were found to be significantly different to those in the untreated samples. This was determined by the t-test and Wilcox-test based on the GSE63159 dataset; as also were the significantly different 25 and 37
DEMs in the GSE45901 dataset for the imatinib-sensitive samples compared to the imatinib-resistant samples. After taking the intersection of these DEMs, a total of 20 DEMs were obtained in the GSE63159 (7 significantly up-regulated and 13 significantly down-regulated) and 23 DEMs in the

A

GSE63159 before normalization

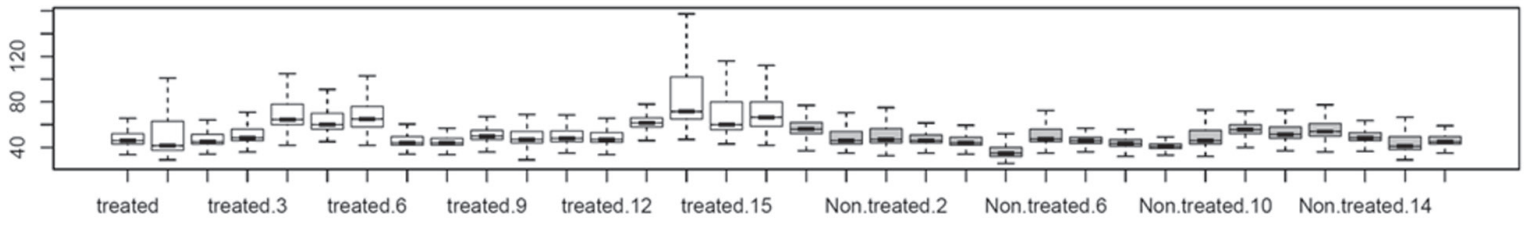

GSE63159 after normalization

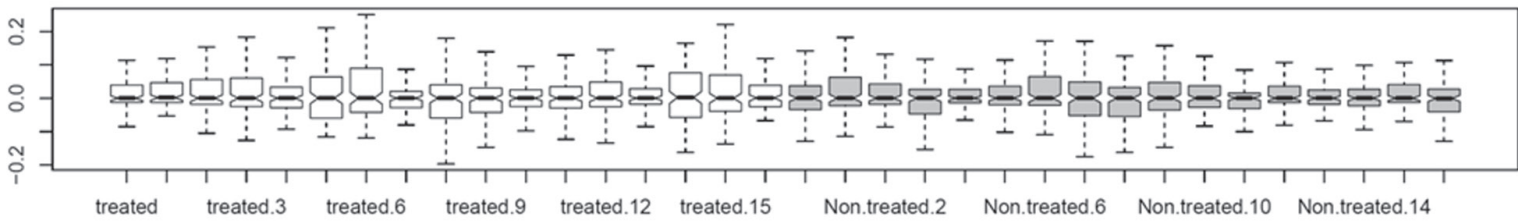

B

GSE45901 before normalization

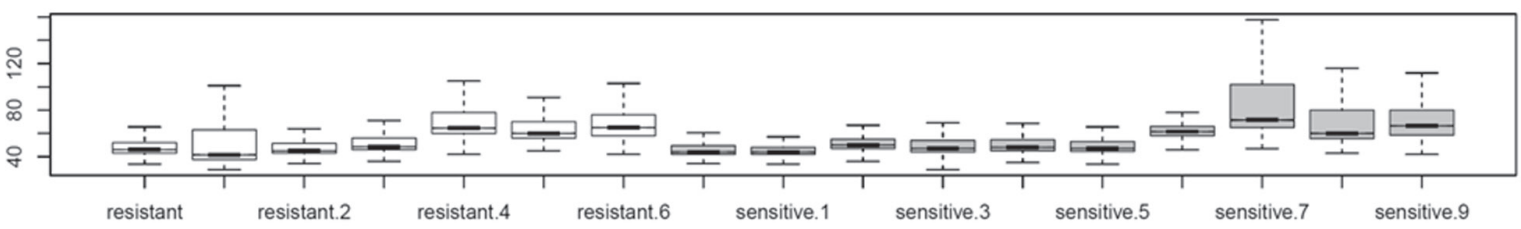

GSE45901 after normalization

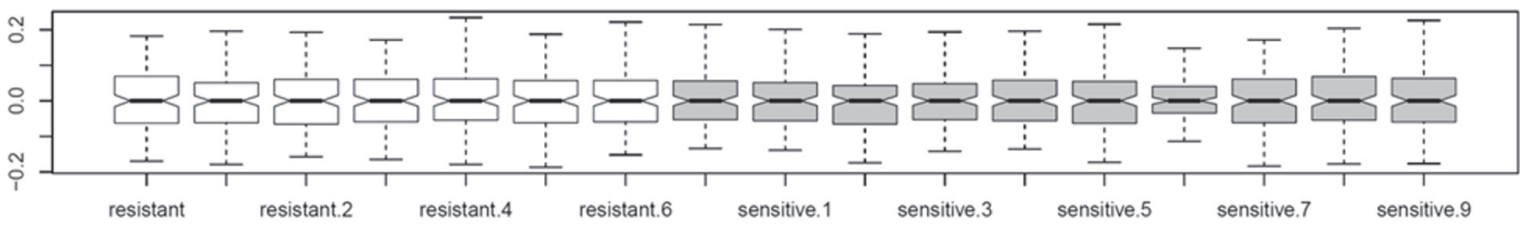

$C_{\text {Student's t test }}$

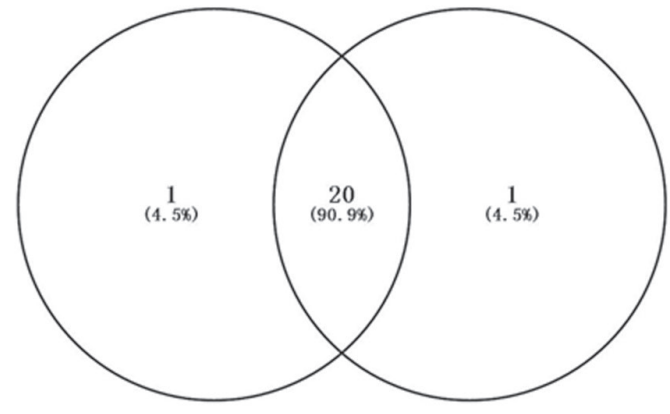

GSE63159

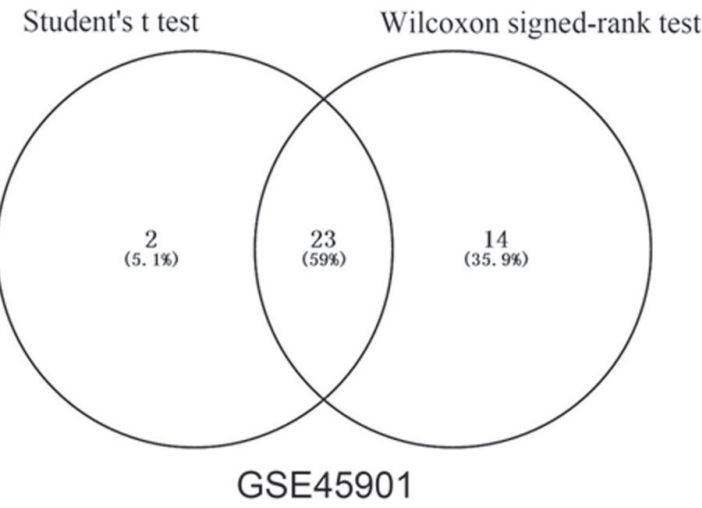

Figure 1. The normalized results of the GSE63159 and GSE45901 datasets. A) The data normalization results from the GSE63159 dataset; B) The data normalization results from the GSE45901 dataset. The white and gray boxes represent imatinib-treated gastrointestinal stromal tumor (GIST) samples and untreated GIST samples, respectively. C) The Venn diagrams show the differentially expressed miRNAs (DEMs) in the GSE63159 and GSE45901 datasets which were examined by t-test and Wilcox-test methods. 
GSE45901 dataset (8 significantly up-regulated and 15 down-regulated). The results are shown in the Figure1C Venn diagram.

Heat map of bidirectional hierarchical clustering analysis. After screening the DEMs in the GSE63159 and GSE45901 datasets, the gene expression values produced a bidirectional hierarchical clustering heat map (Figure 2). The results indicated that DEMs found in the GIST samples could be divided into different groups according to gene expression values.

miRNA-target genes regulatory network construction. DEMs in GSE63159 with a correlation coefficient $>0.8$ were identified. The miRNA-miRNA co-expression network was constructed (Figure 3A). The network included 19 DEMs (5 up-regulated and 14 down-regulated) and 29 edges. These relationships revealed a total of 9 miRNA pairs with negative correlation and 20 pairs with positive correlation.

Based on the results from three datasets, the miRanda, miRTarBase 2016, and TargetScan release 7.1, 52,664, 2487, and 6193 miRNA-target gene pairs were screened, respectively (Figure 3B). In these relationship pairs, 169 miRNAtarget gene pairs existed in the three databases. The miRNAtarget gene regulatory network was then constructed, including 5 critical miRNAs (hsa-miR-324-5p, hsa-miR342-3p, hsa-miR-28-5p, hsa-miR-125a and hsa-miR-320a) and 109 target genes (Figure 3C).

GO function and KEGG pathway enrichment analysis. For further insight into the function of the 109 target genes in the regulatory network, GO function analysis and KEGG pathway enrichment analysis were performed (Figure 4, Table 1). The top twenty functions of these target genes included regulation of cellular protein metabolic processes, protein kinase cascades and cell motion.
KEGG pathway enrichment analysis was performed for these target genes. The target genes were mainly enriched in focal adhesion, the MAPK signaling pathway, neurotrophin signaling pathway, $\mathrm{T}$ cell receptor signaling pathway, axon guidance, leukocyte transendothelial migration, chemokine signaling pathway, adherens junction and the Fc epsilon RI signaling pathway.

Analysis of ceRNA network. A total of 14 lncRNAs were related to $h s a-m i R-125 a-5 p$ expression, including ciliary rootlet coiled-coil, rootletin pseudogene 2 (CROCCP2), chondroitin sulfate proteoglycan 4 pseudogene 5 (CSPG4P5), and Digeorge syndrome critical region gene 5 (DGCR5). In addition, the ceRNA network was constructed. It integrated the relationship of lncRNAs, $h s a-m i R-125 a-5 p$ and target genes. The ceRNAs network in Figure 5A contained 82 nodes (including an miRNA, 14 lncRNAs, and 67 target genes) and 81 edges (including 14 pair lncRNAs-target gene relationships and 67 pair miRNAs-target gene relationships).

GO function and KEGG pathway analysis was performed for the target genes in the ceRNA network. The top 18 functions were involved in three biological processes, eight cell components and seven molecular functions (Figure 5B, Table 2). The biological processes were related to regulation of transcription, DNA-dependent regulation of RNA metabolic processes and intracellular signaling cascades. The cell component relationships included non-membrane-bounded organelles, intracellular non-membrane-bounded organelles and the membrane-enclosed lumen. The molecular functions were related to metal ion binding, cation binding and ion binding. Furthermore, the target genes were mainly enriched in focal adhesion, GnRH signaling pathway, T cell receptor signaling pathway and leukocyte trans-endothelial migration.
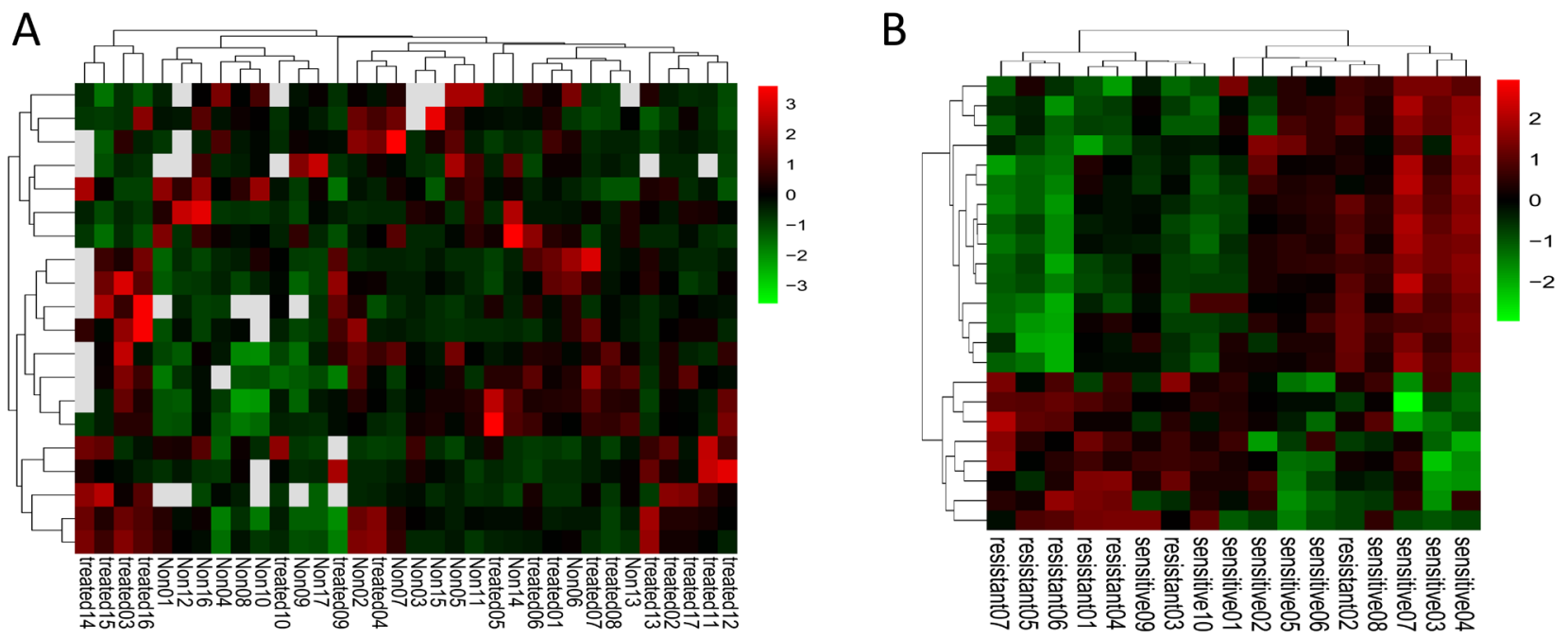

Figure 2. The bidirectional hierarchical clustering heat maps based on the expression value of differentially expressed miRNAs (DEMs) in the GSE63159 (A) and GSE45901 (B) datasets. 

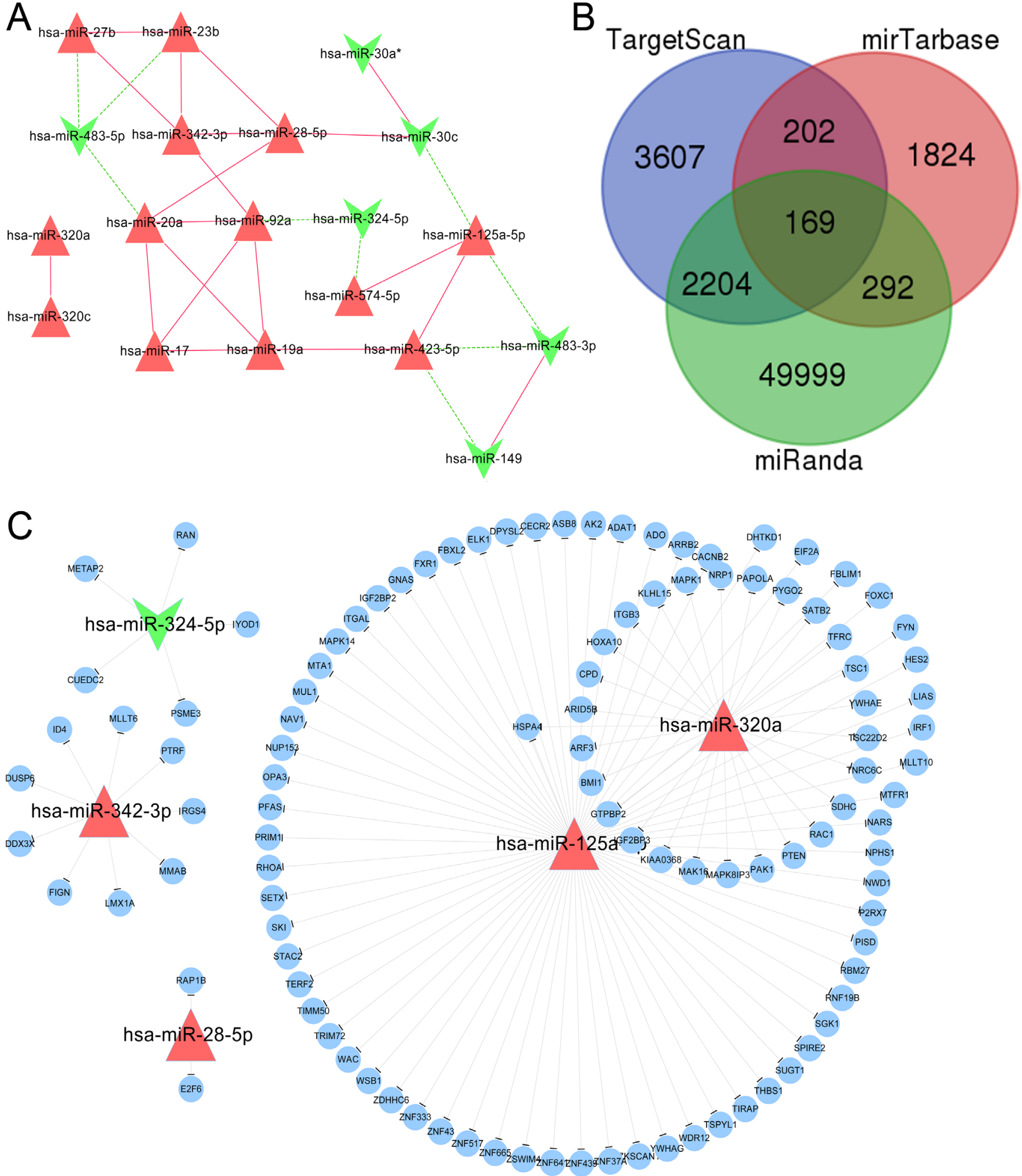

Figure 3. Construction of miRNA co-expression network and miRNA-target gene network. A) The regulatory network of differentially expressed miRNAs (DEMs) in the GSE63159 dataset. Green triangles and red triangles represent significantly down-regulated and up-regulated miRNAs, respectively. B) The Venn diagrams of target genes in three databases, miRanda, miRTarBase, and TargetScan. C) Regulatory network of miRNAs target genes; green triangles and red triangles represent significantly down-regulated and up-regulated miRNA, respectively; blue circular nodes represent target genes. 


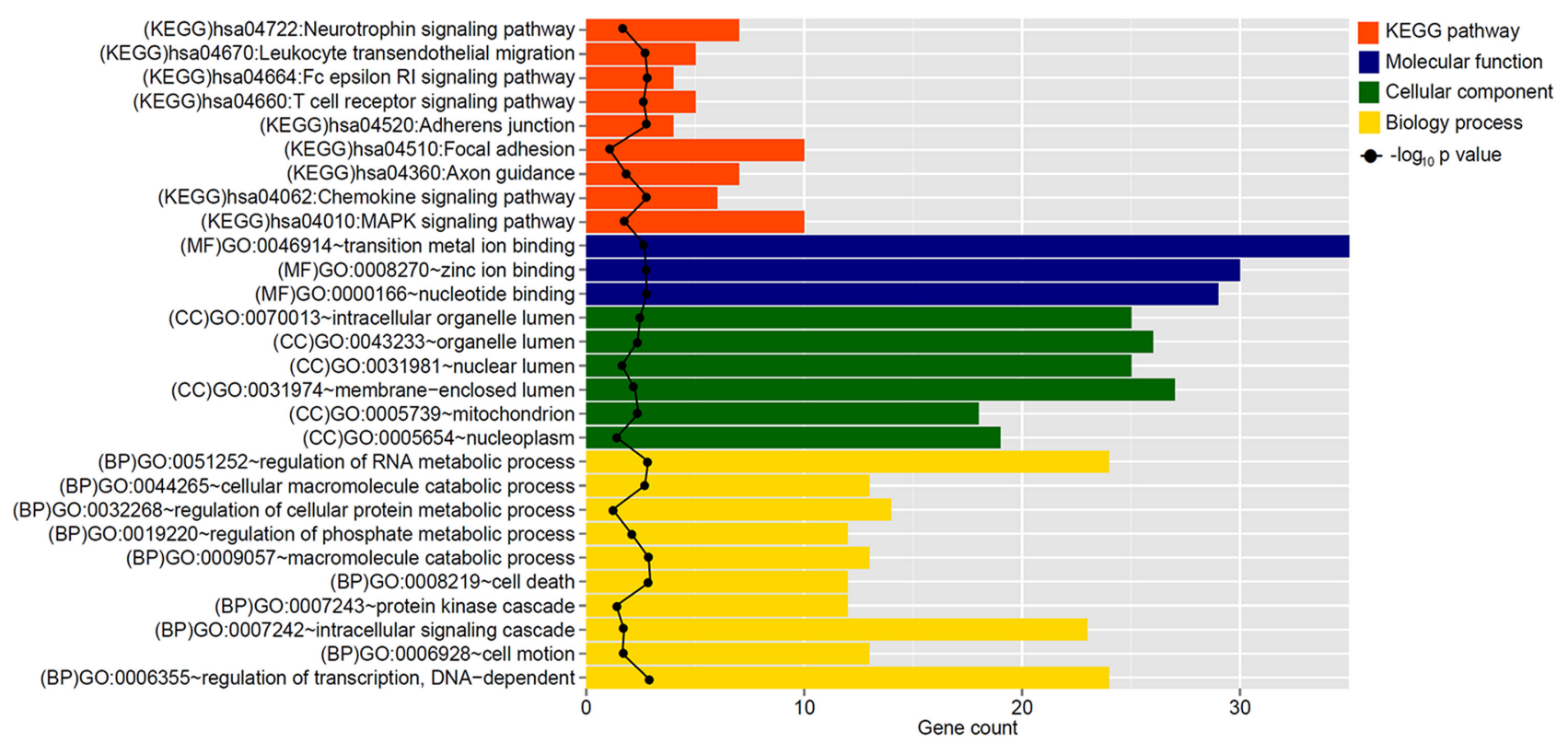

Figure 4. The Gene Ontology (GO) function and Kyoto Encyclopedia of Genes and Genomes (KEGG) pathway enrichment analysis for target genes of miRNAs. Red, blue, green, and yellow represent the KEGG pathway, MF, CC, and BP, respectively; the black line represents the - $\log _{10} p$-value.

Table 1. GO and KEGG pathways for target genes in miRNA-target gene regulation network.

\begin{tabular}{|c|c|c|c|}
\hline Category & Term & Count & p-value \\
\hline \multirow[t]{11}{*}{ Biology Process } & GO:0032268 regulation of cellular protein metabolic process & 14 & $2.48 \mathrm{E}-04$ \\
\hline & GO:0007243 protein kinase cascade & 12 & $3.86 \mathrm{E}-04$ \\
\hline & GO:0006928 cell motion & 13 & $8.94 \mathrm{E}-04$ \\
\hline & GO:0007242 intracellular signaling cascade & 23 & 0.001149 \\
\hline & GO:0019220 regulation of phosphate metabolic process & 12 & 0.003399 \\
\hline & GO:0010605 negative regulation of macromolecule metabolic process & 15 & 0.004701 \\
\hline & GO:0044265 cellular macromolecule catabolic process & 13 & 0.024239 \\
\hline & GO:0006355 regulation of transcription, DNA-dependent & 24 & 0.031822 \\
\hline & GO:0009057 macromolecule catabolic process & 13 & 0.03968 \\
\hline & GO:0051252 regulation of RNA metabolic process & 24 & 0.039732 \\
\hline & GO:0008219 cell death & 12 & 0.04917 \\
\hline \multirow[t]{6}{*}{ Cellular Component } & GO:0005654 nucleoplasm & 19 & $4.48 \mathrm{E}-04$ \\
\hline & GO:0031981 nuclear lumen & 25 & 0.001003 \\
\hline & GO:0031974 membrane-enclosed lumen & 27 & 0.006323 \\
\hline & GO:0043233 organelle lumen & 26 & 0.009575 \\
\hline & GO:0005739 mitochondrion & 18 & 0.010373 \\
\hline & GO:0070013 intracellular organelle lumen & 25 & 0.013847 \\
\hline \multirow[t]{3}{*}{ Molecular Function } & GO:0046914 transition metal ion binding & 35 & 0.028235 \\
\hline & GO:0008270 zinc ion binding & 30 & 0.031614 \\
\hline & GO:0000166 nucleotide binding & 29 & 0.037275 \\
\hline \multirow[t]{9}{*}{ KEGG pathway } & hsa04510:Focal adhesion & 10 & $1.92 \mathrm{E}-04$ \\
\hline & hsa04010:MAPK signaling pathway & 10 & 0.001531 \\
\hline & hsa04722:Neurotrophin signaling pathway & 7 & 0.001674 \\
\hline & hsa04360:Axon guidance & 7 & 0.002049 \\
\hline & hsa04660:T cell receptor signaling pathway & 5 & 0.0254 \\
\hline & hsa04670:Leukocyte transendothelial migration & 5 & 0.033718 \\
\hline & hsa04062:Chemokine signaling pathway & 6 & 0.043995 \\
\hline & hsa04520:Adherens junction & 4 & 0.045564 \\
\hline & hsa04664:Fc epsilon RI signaling pathway & 4 & 0.047047 \\
\hline
\end{tabular}




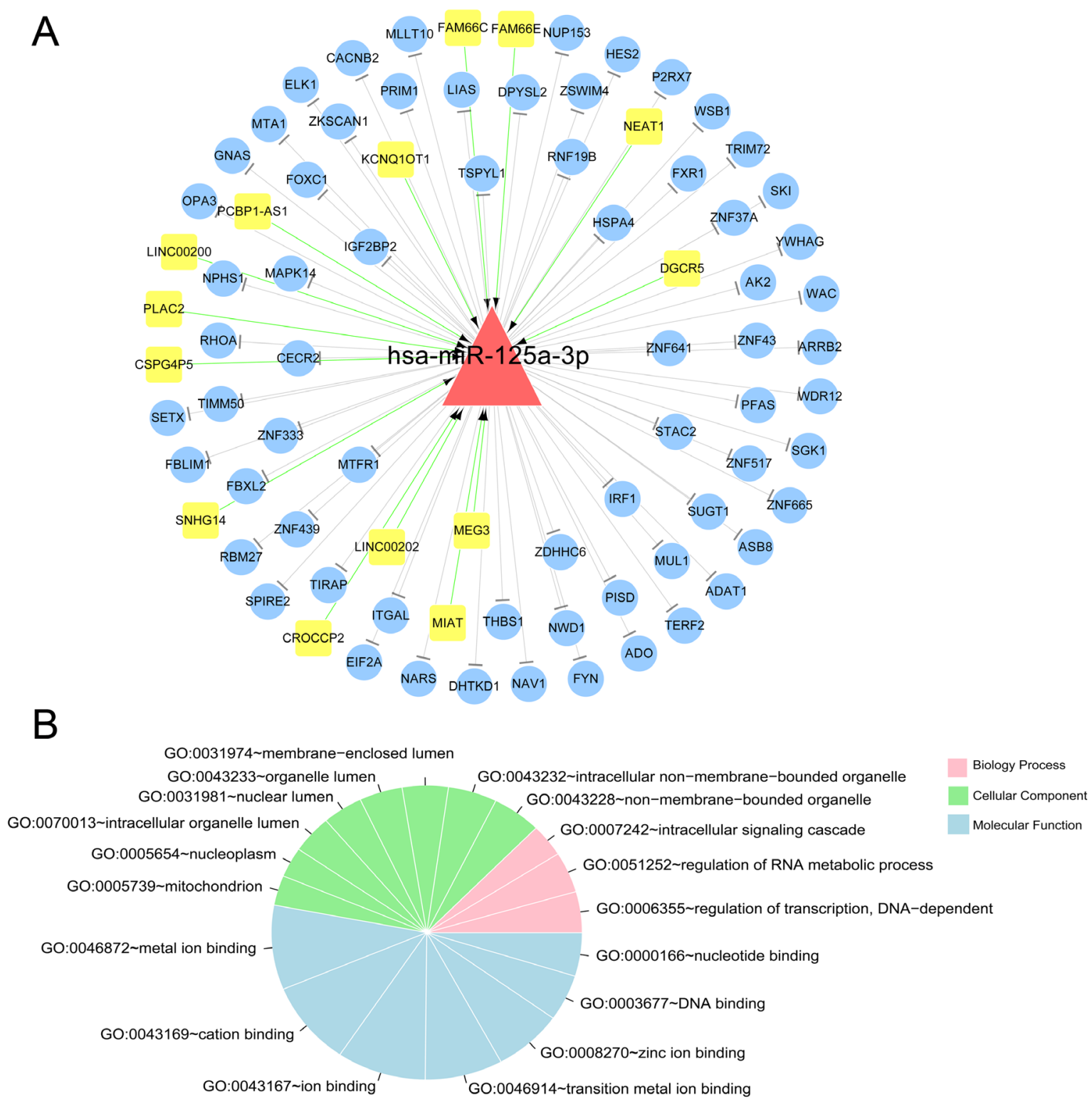

Figure 5. Construction of the ceRNA (IncRNA-miRNA-target gene) network and GO functional annotation diagram of target genes in ceRNA. A) Regulation network of $h s a-m i R-125 a-5 p$ and its target genes. The red triangle represents significantly up-regulated $h s a-m i R-125 a-5 p$, the yellow square node represents IncRNAs, the blue circular node represents target genes, the green arrows represent the relationship between lncRNAs and $h s a-m i R-$ $125 a-5 p$, and the rest show the connection of $h s a-m i R-125 a-5 p$ and its target genes. B) The GO functional annotation diagram of ceRNA. Pink, green, and blue represent $\mathrm{BP}, \mathrm{CC}$, and $\mathrm{MF}$, respectively.

Imatinib resistance analysis in the datasets. Five miRNAs (hsa-miR-574-5p, hsa-miR-125a-5p, hsa-miR-30a*, hsa-miR$30 c$, and hsa-miR-28-5p) were found in the GSE45901 dataset, and these miRNAs overlapped with the DEMs from the GSE63159 dataset. The results indicated that the five miRNAs were critical DEMs in both datasets and related to imatinib-response. Of these miRNAs, $h s a-m i R-28-5 p$ and $h s a-m i R-125 a-5 p$ had significant correlation. Additionally, hsa-miR-125a-5p could be regulated by $14 \operatorname{lncRNAs}$ in a miRNA-target gene regulation network.
To further investigate the function of the hsa-miR-28-5p and $h s a-m i R-125 a-5 p$ critical genes, the expression level of the two miRNAs in the GSE63159 and GSE45901 datasets were analyzed (Figure 6). First, in the GSE63159 dataset, $h s a-m i R-28-5 p$ and $h s a-m i R-125 a-5 p$ were found to be overexpressed in imatinib-treated GIST samples. In the GSE45901 dataset, $h s a-m i R-28-5 p$ and $h s a-m i R-125 a-5 p$ were also overexpressed in imatinib-resistant samples, thus implying that the two miRNAs might be involved in imatinib resistance in GIST patients. 
Table 2. GO and KEGG pathways for target genes in the ceRNA regulation network.

\begin{tabular}{|c|c|c|c|}
\hline Category & Term & Count & p-value \\
\hline \multirow[t]{3}{*}{ Biology Process } & GO:0006355 regulation of transcription, DNA-dependent & 15 & 0.009821 \\
\hline & GO:0051252 regulation of RNA metabolic process & 15 & 0.011867 \\
\hline & GO:0007242 intracellular signaling cascade & 12 & 0.011145 \\
\hline \multirow[t]{8}{*}{ Cellular Component } & GO:0043228 non-membrane-bounded organelle & 17 & 0.009949 \\
\hline & GO:0043232 intracellular non-membrane-bounded organelle & 17 & 0.009949 \\
\hline & GO:0031974 membrane-enclosed lumen & 16 & $9.09 \mathrm{E}-04$ \\
\hline & GO:0043233 organelle lumen & 15 & 0.002327 \\
\hline & GO:0031981 nuclear lumen & 14 & $8.61 \mathrm{E}-04$ \\
\hline & GO:0070013 intracellular organelle lumen & 14 & 0.005489 \\
\hline & GO:0005654 nucleoplasm & 11 & $6.69 \mathrm{E}-04$ \\
\hline & GO:0005739 mitochondrion & 11 & 0.003246 \\
\hline \multirow[t]{7}{*}{ Molecular Function } & GO:0046872 metal ion binding & 31 & 4.10E-04 \\
\hline & GO:0043169 cation binding & 31 & $4.91 \mathrm{E}-04$ \\
\hline & GO:0043167 ion binding & 31 & $6.49 \mathrm{E}-04$ \\
\hline & GO:0046914 transition metal ion binding & 27 & $1.80 \mathrm{E}-05$ \\
\hline & GO:0008270 zinc ion binding & 24 & $2.70 \mathrm{E}-05$ \\
\hline & GO:0003677 DNA binding & 17 & 0.028924 \\
\hline & GO:0000166 nucleotide binding & 16 & 0.042922 \\
\hline \multirow[t]{4}{*}{ KEGG pathway } & hsa04510:Focal adhesion & 4 & 0.046024 \\
\hline & hsa04912:GnRH signaling pathway & 3 & 0.047158 \\
\hline & hsa04660:T cell receptor signaling pathway & 3 & 0.04847 \\
\hline & hsa04670:Leukocyte transendothelial migration & 3 & 0.04985 \\
\hline
\end{tabular}

\section{Discussion}

In this study, we identified the $h s a-m i R-28-5 p$ and hsa-miR-125a-5p were associated with imatinib resistance or imatinib sensitivity in GIST patients. GO function and KEGG pathway analysis showed that the two miRNAs regulated GIST progression through focal adhesion and the GnRH signaling pathway.

Previous studies have revealed that miRNAs play an important role in tumor pathogenesis, invasion and drug resistance in cancers [24-26], and Akakaya P [13] showed that over-expression of $h s a-m i R-125 a-5 p$ was associated with imatinib resistance in GIST patients; the potential mechanism is that $h s a-m i R-125 a-5 p$ can increase cell viability by down-regulating the expression level of PTPN18 in tumor cells. Zheng et al. [27] showed that down-regulation of several miRNAs, including $h s a-m i R-125 a-5 p$ correlated with poor prognosis in gastrointestinal cancer patients. Herein, hsa-miR-125a-5p was overexpressed in imatinib-treated GIST samples, suggesting that $h s a-m i R-125 a-5 p$ functions as a major regulator in the progression of GIST.

KEGG pathway enrichment analysis showed that the $h s a-m i R-125 a-5 p$ target genes were mainly enriched in focal adhesion and GnRH signaling pathways. Focal adhesion has been confirmed to play an important role in tumor metastasis [28]. Murata et al. [29] showed that focal adhesion kinase (FAK) was an important mediator, functioning between cells and the extracellular matrix and that FAK localization was related to colorectal carcinogenesis. Wang et al. [30] reported that mutations of Rho GTPases might affect the progression of gastric cancer through adherent junction and focal adhesion pathway, and a recent study [31] revealed that silencing profilin-1 could inhibit gastric cancer progression, with the effect most likely mediated by the integrin $\beta 1 /$ FAK pathway. In addition, the GnRH signaling pathway was reported to be involved in tumor pathogenesis and progression in several cancers, such as lung, pancreatic and colon cancer [32].

Further, GnRH agonists have been demonstrated to inhibit ovarian, prostate and breast cancer cell progression [33-35]. GnRH might also significantly decrease angiogenesis in melanoma by regulating the VEGFs pathway [36]. However, the effect of the GnRH signaling pathway in GIST was less reported. Chang et al. [37] identified multiple novel genes associated with metastasis of gastric cancer. Based on our findings, we speculate that $h s a-m i R-125 a-5 p$ might play an important role in GIST progression and that the effect of the imatinib-response was likely mediated through the focal adhesion and GnRH signaling pathways. However, the potential mechanism of $h s a-m i R-125 a-5 p$ in imatinib resistance in GIST still needs further investigation.

Although $h s a-m i R-28-5 p$ was also over-expressed in drug resistant GIST samples, very little is known about it except that its up-regulation was reported in ovarian cancer tissues compared to adjacent ovarian tissues, and it might promote ovarian tumor growth through down-regulation of N4BP1 in vivo [38]. Almeida et al. [7] also revealed 
A

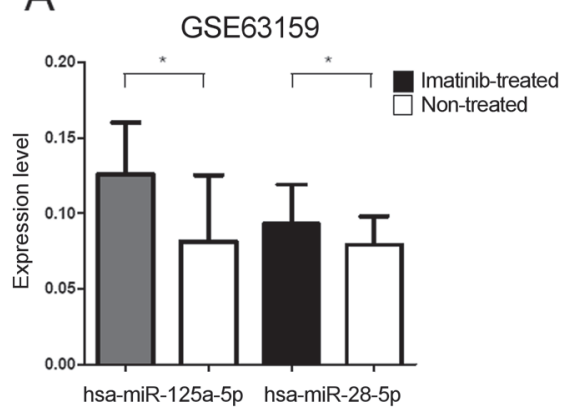

$\mathrm{B}$

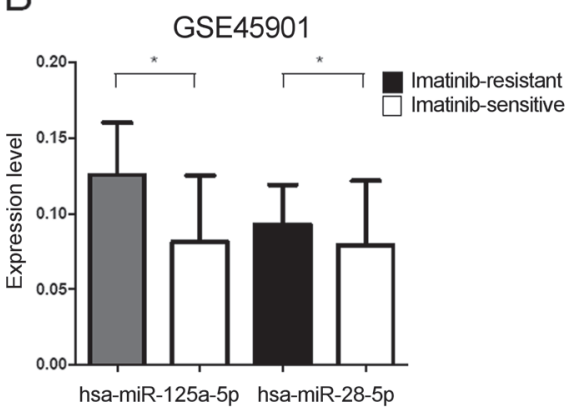

Figure 6. The expression level of $h s a-m i R-125 a-5 p$ and $h s a-m i R-28-5 p$ in the GSE63159 (A) and GSE45901 (B) datasets. A) The gray and black columns represent the expression level of $h s a-m i R-125 a-5 p$ and $h s a-m i R-28-5 p$ in imatinib-treated GIST samples, respectively and the white columns represent the untreated GIST samples. B) The gray and black columns represent the expression level of $h s a-m i R-125 a-5 p$ and $h s a-m i R-28$-5 $p$ in imatinib resistance GIST samples, respectively, while the white columns represent the imatinib-sensitive GIST samples. ${ }^{*}$ indicates significant difference between the two groups $(\mathrm{p}<0.05)$.

that $a-m i R-28-5 p$ suppressed tumor cell proliferation and caused tumor cell apoptosis in colorectal cancer cells.

Although the function of $h s a-m i R-28-5 p$ in GIST progress has not been reported, we speculate that over-expression of hsa-miR-28-5p may play an important role in imatinib resistance in GIST patients.

The strengths of this study include the identification of $h s a-m i R-28-5 p$ and $h s a-m i R-125 a-5 p$ which provides us with a broader perspective for elucidating the key mechanism associated with imatinib resistance and sensitivity in imatinib-treated GIST patients.

However, there are some limitations that merit further consideration. Firstly, the expression levels of the identified key miRNAs were not verified by performing biological experiments with patient samples. Secondly, our experiments did not exclusively confirm if changing the expression of these two miRNAs can alter the sensitivity to imatinib treatment, and therefore further experiments are required to confirm the findings of this study.

In conclusion, our study based on bioinformatics analysis suggests that the critical miRNAs, such as $h s a-m i R-28-5 p$ and $h s a-m i R-125 a-5 p$ are most likely associated with imatinib resistance or sensitivity in imatinib-treated GIST patients. $H s a-m i R-28-5 p$ and $h s a-m i R-125 a-5 p$ may also be considered prognostic markers in imatinib-response in GIST patients.

Acknowledgments: This study was supported by the Scientific Research Project in Heilongjiang Province, [Project No. 201713].

\section{References}

[1] NILSSON B, BUMMING P, MEIS-KINDBLOM JM, ODEN A, DORTOK A et al. Gastrointestinal stromal tumors: the incidence, prevalence, clinical course, and prognostication in the preimatinib mesylate era--a population-based study in western Sweden. Cancer 2005; 103: 821-829. https://doi. org/10.1002/cncr.20862
[2] HIROTA S, ISOZAKI K, MORIYAMA Y, HASHIMOTO $\mathrm{K}$, NISHIDA T et al. Gain-of-function mutations of c-kit in human gastrointestinal stromal tumors. Science 1998; 279: 577-580.

[3] DUENSING S, DUENSING A. Targeted therapies of gastrointestinal stromal tumors (GIST)--the next frontiers. Biochem Pharmacol 2010; 80: 575-583. https://doi. org/10.1016/j.bcp.2010.04.006

[4] JOENSUU H, DEMATTEO RP. The management of gastrointestinal stromal tumors: a model for targeted and multidisciplinary therapy of malignancy. Annu Rev Med 2012; 63: 247-258. https://doi.org/10.1146/annurevmed-043010-091813

[5] CORLESS CL, MCGREEVEY L, HALEY A, TOWN A, HEINRICH MC. KIT mutations are common in incidental gastrointestinal stromal tumors one centimeter or less in size. Am J Pathol 2002; 160: 1567-1572. https://doi.org/10.1016/ S0002-9440(10)61103-0

[6] ZHANG J, CHENG J, ZENG Z, WANG Y, LI X et al. Comprehensive profiling of novel microRNA-9 targets and a tumor suppressor role of microRNA-9 via targeting IGF2BP1 in hepatocellular carcinoma. Oncotarget 2015; 6: 4204042052. https://doi.org/10.18632/oncotarget.5969

[7] ALMEIDA MI, NICOLOSO MS, ZENG L, IVAN C, SPIZZO R et al. Strand-specific miR-28-5p and miR-28-3p have distinct effects in colorectal cancer cells. Gastroenterology 2012; 142: 886-896 e889. https://doi.org/10.1053/j.gastro.2011.12.047

[8] SUBRAMANIAN S, LUI WO, LEE CH, ESPINOSA I, NIELSEN TO et al. MicroRNA expression signature of human sarcomas. Oncogene 2008; 27: 2015-2026. https://doi. org/10.1038/sj.onc. 1210836

[9] KIM WK, YANG HK, KIM H. MicroRNA involvement in gastrointestinal stromal tumor tumorigenesis. Curr Pharm Des 2013; 19: 1227-1235.

[10] NIINUMA T, SUZUKI H, NOJIMA M, NOSHO K, YAMAMOTO H et al. Upregulation of miR-196a and HOTAIR drive malignant character in gastrointestinal stromal tumors. Cancer Res 2012; 72: 1126-1136. https://doi.org/10.1158/00085472.CAN-11-1803 
[11] NANNINI M, BIASCO G, ASTOLFI A, PANTALEO MA. An overview on molecular biology of KIT/PDGFRA wild type (WT) gastrointestinal stromal tumours (GIST). J Med Genet 2013; 50: 653-661. https://doi.org/10.1136/jmedgenet-2013-101695

[12] AKCAKAYA P, LUI WO. MicroRNAs and Gastrointestinal Stromal Tumor. Adv Exp Med Biol 2015; 889: 51-70. https:// doi.org/10.1007/978-3-319-23730-5_4

[13] AKCAKAYA P, CARAMUTA S, AHLEN J, GHADERI M, BERGLUND E et al. microRNA expression signatures of gastrointestinal stromal tumours: associations with imatinib resistance and patient outcome. Br J Cancer 2014; 111: 20912102. https://doi.org/10.1038/bjc.2014.548

[14] YOSHIDA R, IMOTO S, HIGUCHI T. Estimating time-dependent gene networks from time series microarray data by dynamic linear models with Markov switching. Proc IEEE Comput Syst Bioinform Conf 2005: 289-298.

[15] GAUTIER L, COPE L, BOLSTAD BM, IRIZARRY RA. affy-analysis of Affymetrix GeneChip data at the probe level. Bioinformatics 2004; 20: 307-315. https://doi.org/10.1093/ bioinformatics/btg405

[16] WANG L, CAO C, MA Q, ZENG Q, WANG H et al. RNA-seq analyses of multiple meristems of soybean: novel and alternative transcripts, evolutionary and functional implications. BMC Plant Biol 2014; 14: 169. https://doi.org/10.1186/14712229-14-169

[17] DEZA MM, DEZA E. (Eds.). Encyclopedia of Distances. Springer-Verlag Berlin, 2009, p. 583. ISBN 978-3-642-00233-5

[18] SZEKELY G, RIZZO, ML. Hierarchical Clustering via Joint Between-Within Distances: Extending Ward's Minimum Variance Method. Journal of Classification 2005; 22: 151183.

[19] SHANNON W, CULVERHOUSE R, DUNCAN J. Analyzing microarray data using cluster analysis. Pharmacogenomics 2003; 4: 41-52. https://doi.org/10.1517/phgs.4.1.41.22581

[20] BETEL D, KOPPAL A, AGIUS P, SANDER C, LESLIE C. Comprehensive modeling of microRNA targets predicts functional non-conserved and non-canonical sites. Genome Biol 2010; 11: R90. https://doi.org/10.1186/gb-2010-118-r90

[21] CHOU CH, CHANG NW, SHRESTHA S, HSU SD, LIN YL et al. miRTarBase 2016: updates to the experimentally validated miRNA-target interactions database. Nucleic Acids Res 2016; 44: D239-247. https://doi.org/10.1093/nar/ gkv1258

[22] AGARWAL V, BELL GW, NAM JW, BARTEL DP. Predicting effective microRNA target sites in mammalian mRNAs. Elife 2015; 4. https://doi.org/10.7554/eLife.05005

[23] SHANNON P, MARKIEL A, OZIER O, BALIGA NS, WANG JT et al. Cytoscape: a software environment for integrated models of biomolecular interaction networks. Genome Res 2003; 13: 2498-2504. https://doi.org/10.1101/gr.1239303

[24] SARKAR FH, LI Y, WANG Z, KONG D, ALI S. Implication of microRNAs in drug resistance for designing novel cancer therapy. Drug Resist Updat 2010; 13: 57-66. https://doi. org/10.1016/j.drup.2010.02.001
[25] CALIN GA, SEVIGNANI C, DUMITRU CD, HYSLOP T, $\mathrm{NOCH}$ E et al. Human microRNA genes are frequently located at fragile sites and genomic regions involved in cancers. Proc Natl Acad Sci U S A 2004; 101: 2999-3004. https:// doi.org/10.1073/pnas.0307323101

[26] ZHANG B, PAN X, COBB GP, ANDERSON TA. microRNAs as oncogenes and tumor suppressors. Dev Biol 2007; 302: 1-12. https://doi.org/10.1016/j.ydbio.2006.08.028

[27] ZHENG Q, CHEN C, GUAN H, KANG W, YU C. Prognostic role of microRNAs in human gastrointestinal cancer: A systematic review and meta-analysis. Oncotarget 2017; 8: 46611-46623. https://doi.org/10.18632/oncotarget.16679

[28] ZAMIR E, GEIGER B. Molecular complexity and dynamics of cell-matrix adhesions. J Cell Sci 2001; 114: 3583-3590.

[29] MURATA T, NAOMOTO Y, YAMATSUII T, OKAWA T, SHIRAKAWA Y et al. Localization of FAK is related with colorectal carcinogenesis. Int J Oncol 2008; 32: 791-796.

[30] WANG K, YUEN ST, XU J, LEE SP, YAN HH et al. Wholegenome sequencing and comprehensive molecular profiling identify new driver mutations in gastric cancer. Nat Genet 2014; 46: 573-582. https://doi.org/10.1038/ng.2983

[31] CHENG YJ, ZHU ZX, ZHOU JS, HU ZQ, ZHANG JP et al. Silencing profilin-1 inhibits gastric cancer progression via integrin betal/focal adhesion kinase pathway modulation. World J Gastroenterol 2015; 21: 2323-2335. https://doi. org/10.3748/wjg.v21.i8.2323

[32] NEILL JD. GnRH and GnRH receptor genes in the human genome. Endocrinology 2002; 143: 737-743. https://doi. org/10.1210/endo.143.3.8705

[33] DONDI D, LIMONTA P, MORETTI RM, MARELLI MM, GARATTINI E et al. Antiproliferative effects of luteinizing hormone-releasing hormone (LHRH) agonists on human androgen-independent prostate cancer cell line DU 145: evidence for an autocrine-inhibitory LHRH loop. Cancer Res 1994; 54: 4091-4095.

[34] KERI G, BALOGH A, SZOKE B, TEPLAN I, CSUKA O. Gonadotropin-releasing hormone analogues inhibit cell proliferation and activate signal transduction pathways in MDA-MB-231 human breast cancer cell line. Tumour Biol 1991; 12: 61-67.

[35] GRUNDKER C, GUNTHERT AR, WESTPHALEN S, EMONS G. Biology of the gonadotropin-releasing hormone system in gynecological cancers. Eur J Endocrinol 2002; 146: $1-14$.

[36] MORETTI RM, MAI S, MONTAGNANI MARELLI M, BANI MR, GHILARDI C et al. Dual targeting of tumor and endothelial cells by gonadotropin-releasing hormone agonists to reduce melanoma angiogenesis. Endocrinology 2010; 151: 4643-4653. https://doi.org/10.1210/en.2010-0163

[37] CHANG W, MA L, LIN L, GU L, LIU X et al. Identification of novel hub genes associated with liver metastasis of gastric cancer. Int J Cancer 2009; 125: 2844-2853. https://doi. org/10.1002/ijc.24699

[38] XU J, JIANG N, SHI H, ZHAO S, YAO S et al. miR-28-5p promotes the development and progression of ovarian cancer through inhibition of N4BP1. Int J Oncol 2017; 50: 13831391. https://doi.org/10.3892/ijo.2017.3915 